\title{
Computer Algorithm for Creation Classic Mosaic Fillings Based on the Vector Guide Lines
}

\author{
Andri Petrushevski, Victoriia Us
}

\begin{abstract}
The article suggests algorithms for filling mosaic surfaces. Today, there are a number of algorithms that use the software design environment to automate the creation of images that correspond to mosaic compositions based on raster images. However, they do not allow realizing the principles of the classical construction of mosaic works, which guide professional mosaics. In this case, the main task is the problem of optimizing the filling of space from a mathematical point of view. From the point of view of the artist, this is not the main problem, moreover, the distance between the parts of the mosaic can vary, act as an artistic element. Much more important is the organization of the structure of the mosaic surface. This article is dedicated to this problem. The sequence of actions is given - algorithms that allow the artist to achieve the desired effect and gain full control when filling mosaic surfaces. These algorithms allow you to create mosaic fillings based on linear patterns without color and tonal design, which is impossible for existing algorithms. The authors proposed the construction of the topology of classical mosaics using vector guides. When forming the algorithm, vector guide lines or "power lines" are taken as a basis, on which mosaic elements (tesserae) are subsequently placed. Variants of using existing algorithms at each stage of the solution of the problem are proposed. Algorithms and results of filling a mosaic panel with a line-by-line and equidistant method are presented. This technology will allow not only creating mosaic surfaces, but also using it as a tool for creating real art projects for implementation in design and art. The possibility of using existing technologies in this technique is commented.
\end{abstract}

Keywords: mosaic, tesserae, guiding lines of force, filling algorithms, contour areas.

\section{INTRODUCTION}

Mosaic is perhaps one of the most ancient forms of art. Numerous works of fine art of the past and the present are made using the mosaic technique. World famous masterpieces of the ancient Greek, Roman, Byzantine period and many author panels of the late time. Despite the fact that we all admire the work of the past, today less and less artists resort to the classical methods of performing works. And

Revised Manuscript Received on February 05, 2020.

* Correspondence Author

Andri Petrushevski*. State university of infrastructure and technologies, Department of Information Technology, Kyiv, Ukraine. Email: zmodeller@gmail.com

Victoriia Us. National Aviation University, Interior Design department, associate professor, Kyiv, Ukraine. Email: yc0869@gmail.com

(c) The Authors. Published by Blue Eyes Intelligence Engineering and Sciences Publication (BEIESP). This is an open access article under the CC BY-NC-ND license (http://creativecommons.org/licenses/by-nc-nd/4.0/) mosaic, in this regard, is no exception. Blame this on contemporary artists. As with any phenomenon, there is a logical explanation. Digital tools are more convenient, provide more features, reduce execution time and provide a number of additional features. This is facilitated by the changing habits of society. The principles of aesthetics and harmony have not been canceled. Only from an ergonomic point of view, unsuitable color, sound combinations and compositional solutions can lead to psychological and even physiological discomfort of the user. For thousands of years, classical art has shaped the principles and methods of constructing a work. During this time, it has accumulated a lot of knowledge and practical techniques. Already during the 20th century, even before the digital age, unfortunately, there was a great loss of the school in the field of mosaic art technology. And in our time, the few who have preserved the classical school, in most cases, are not able to adapt to modern changes. Representatives of the young generation, who thoroughly study digital art technologies, often do not devote enough time to studying classical principles, thereby creating their own subculture. Moreover, often, for one reason or another, those involved in software development do not pay attention to this at all. Thus, the user gets his hands on tools that do not take into account many factors. This article is an attempt to draw the attention of specialists working on the creation of computer image algorithms to some principles of constructing classical mosaic fillings. Algorithms are proposed that make it possible, according to the authors, to resemble the classic mosaic styles.

\section{BASIC ASPECTS OF MOSAIC PANEL FILLING ALGORITHMS}

The graphic filter "mosaic", well known for a large number of computer programs (Adobe Photoshop, GIMP, Adobe Illustrator and others), which provide a decrease in spatial resolution (Fig. 1). OF COURSE, THE DEVELOPERS OF SUCH A FILTER SAW THIS PROBLEM. TO SIMULATE MORE "ARTISTRY" SEVERAL PARAMETERS HAVE BEEN ADDED TO THE ALGORITHM; SLIGHT CURVATURE OF THE LINES, THE DISTANCE BETWEEN THE TEASERS AND COLOR VARIATIONS. IT IS CLEAR THAT WITH THESE MEANS NO ONE CLAIMED TO CREATE A REAL STYLE.

This arrangement of teasers for forming a closed plane does not correspond to any of the artistic means. 


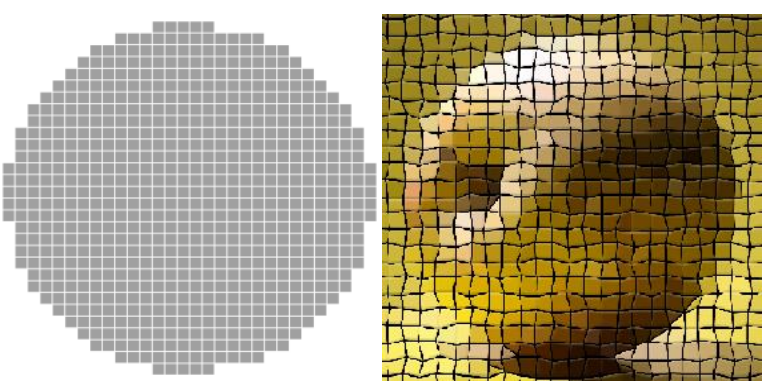

Fig. 1.

The classic mosaic canvas has a certain structure, which in turn depends on the composition of the geometric shapes depicted on the plane. It is the form of this structure that the styles differ.

A number of basic historical styles of mosaics are known: the Greek style, the Byzantine style and the Florentine style.

In the scientific literature there are a number of developments that are interesting mathematical solutions for the formation of mosaic images mainly built on Voronoi diagrams [1]. It remains a mystery why the leading manufacturers of software for artists did not take advantage of these developments. But all developers have one drawback. They do not take into account the real methods of creating the structure of the mosaic work and are difficult to control. Every professional specialist in the field of mosaics knows that drawing is the basis of any mosaic work. The drawing is created taking into account the specifics of the material. A certain kind of stylization of the form is performed. The pattern is divided into spots that are delimited by the edges of the form and the shadow lines in the case of volumes. Further, each spot is filled with the so-called "power lines". The direction of these lines is built taking into account the movement of the shape of the object. Directly, a set of mosaic filling is performed according to a pattern created in advance. Tesserae is laid out along these lines. The artist does this in accordance with his experience. We can create a special algorithm for filling the surface. But the principle itself must be preserved. If you look closely at the classic works of mosaic art, you can see that even the background has specific patterns of filling.

Consider the three main stages in the formation of algorithms.

1. Fill the background.

To fill the background, you can use specialized vector libraries of historically formed patterns in the form of vector clipart, for example, in the SVG format (Fig. 2). The user will be able to choose a suitable pattern in accordance with the style of his work. Consider the three main stages in the formation of algorithms.

1. Fill the background.

To fill the background, you can use specialized vector libraries of historically formed patterns in the form of vector clipart, for example, in the SVG format (Fig. 2). The user will be able to choose a suitable pattern in accordance with the style of his work. Not one of the developed algorithms for automated filling will not give us such an opportunity. Especially in decorative and applied works. Further, having received the contours of the elements, we can remove them from the background using logical operations.

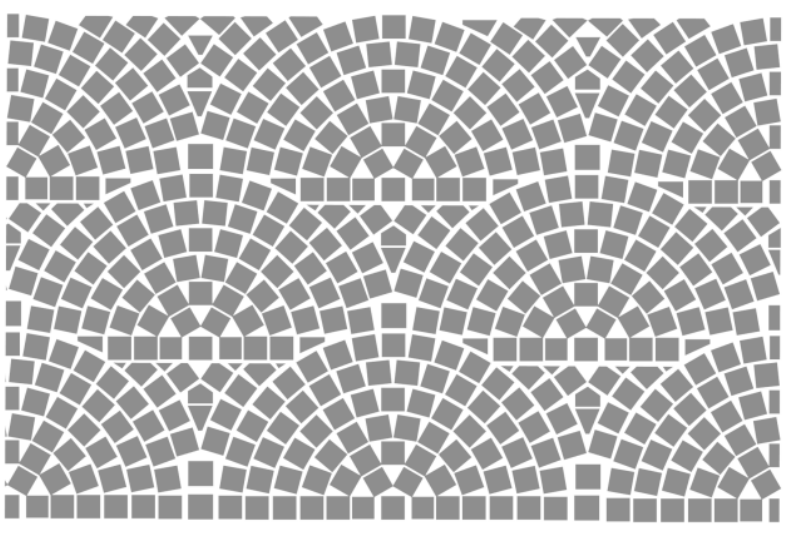

Fig. 2.

2. Contour drawing of the image.

As a rule, raster images are used to create mosaic fillings with computer filters. It can be sketches or photos. To solve our problem of image contour rendering, we perform vector image tracing to obtain a linear picture based on contrasting pixel arrays (Fig. 3).
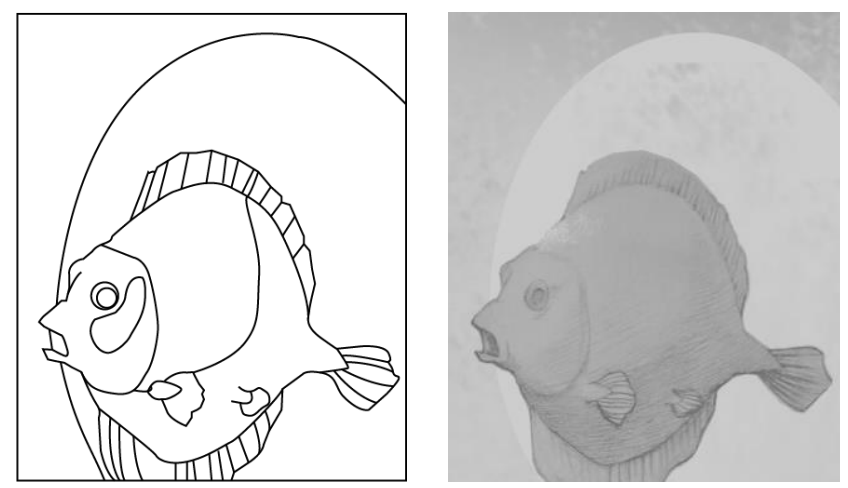

Fig. 3.

There are a large number of ready-made linear and polygonal vectorization algorithms [2]. In our case, it is possible to use any linear vectorization algorithm based on thinning or based on the contours of R2V, MIC, SPV, LNG [2]. If the image is sufficiently contrasted, then there should be no problems. If the tone of the image is identical, operator intervention is required. The user will have to correct the incorrectly drawn lines. To get a stylistically believable image, we can't avoid operator intervention. Alternatively, specialized artificial intelligence (AI) algorithms can be used to correct problem areas [3]. Corrections may be needed not only in terms of erroneous directions. Artistic edits are possible. Alternatively, you can give the user various options for the vectored image created by the AI algorithm to choose from. Thus, we obtain the area divided into closed areas.

3. Filling closed areas.

Each of the obtained closed areas must be filled with mosaic parts in a certain way. There can be four main ways to fill closed areas of a mosaic.

1. Line by line. The contour area is filled with mosaic parts as lines in the text in one selected direction. Perhaps a straight or arbitrary curvature of the lines (wavy) (Fig. 4).

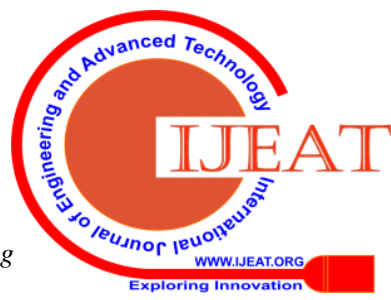



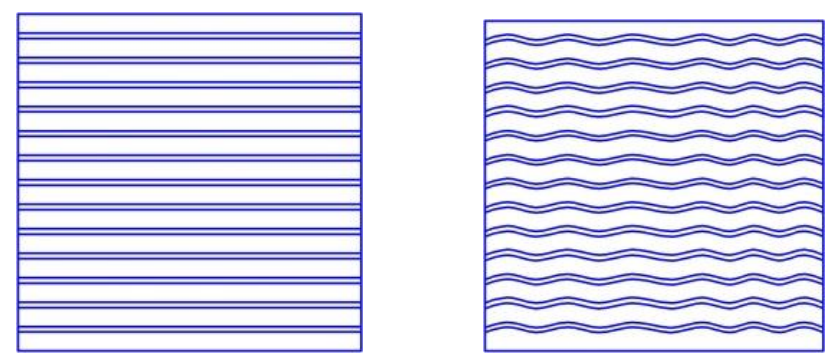

Fig. 4.

2. Equidistant, in the form of concentric circles, ellipses, with rectilinear or wavy contours. The selected contour region is duplicated to the center with a decrease at an equal distance (Fig. 5).

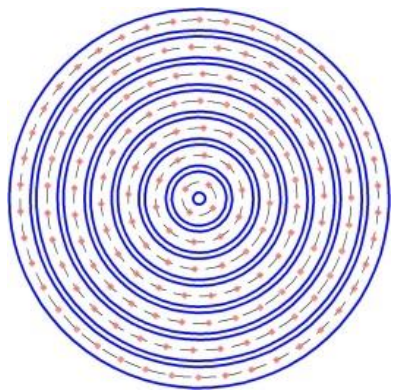

Fig. 5.

3. Spiral. The selected contour region is filled with parts of the mosaic along the spiral guide to the center of the contour region, with rectilinear or wavy contours (Fig. 6).

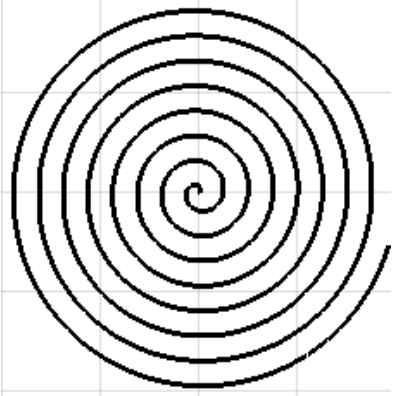

Fig. 6.

4. Combined. The selected contour area is filled in a mixed way, consisting of various combinations of the first three methods.

The main algorithms for filling contour selected areas are:

1. A method based on Voronoi diagrams (CVD) [4].

2. Irregular tiles based on gradient vector flow (GVF) [5].

3. Jigsaw Image Mosaic algorithm [6].

For use in visualization of classic mosaic styles, it is advisable to use the first two algorithms. These algorithms are designed for automatic execution. With this approach, it is difficult to control the topology of each region. However, these algorithms are ideal for filling contour areas in the third way. Some unpredictability of the result will even be useful and give vividness to such a surface.

When forming the algorithm, we take vector guide lines or "power lines" as a basis, on which mosaic elements (tesserae) are subsequently placed (Fig. 7).

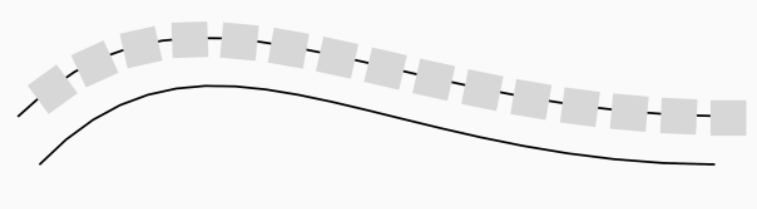

Fig. 7.

Line-by-line method of forming an algorithm.

This method is the simplest in execution.

If the body, which is filled with a mosaic, has a shape close to the shape of a square, rectangle or trapezoid, use the linear (straight or wavy lines) arrangement of teasers on them (Fig. 4).

With a linear arrangement of teasers to form a mosaic, we use the following algorithm:

Determine the linear dimensions of the mosaic canvas: width - $A$ and height - $B$;

Choose the size of the side of the teaser - $m$ and the size of the seam - $p$;

Determine the distance between the centers of the teasers $d=m+p$ (Fig. 8).

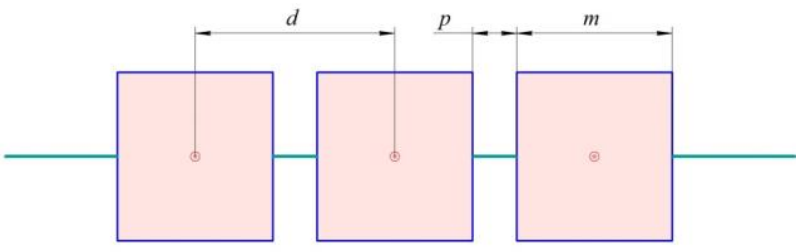

Fig. 8.

Determine the number of lines for placing teasers horizontally

$$
k=\frac{A}{d}
$$

Determine the number of lines for placing teasers vertically

$$
n=\frac{B}{d}
$$

We are building the mosaic.

If the line on which the tesserae are curved or wavy, their direction is determined by the shape of the contour area. That is, to what geometric primitive the contour region will be approximated. For example, a triangle, rectangle, circle, or ellipse. In this case, you first need to approximate the shape of the contour selected area. For this, you can use existing algorithms, for example, a multivariate method for recognizing patterns and geometric shapes [7]. In this case, temporary adjacency and global geometric properties of figures are used to recognize a simple dictionary of geometric shapes, including solid and dashed line styles. The geometric elements used (convex body, regular polygons of the smallest area, scalar relations of the perimeter and area) are invariant with the rotation and scale of the figures.

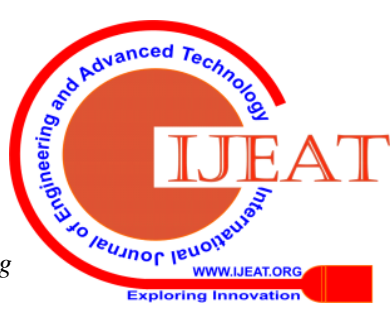


If we consider a form that is close to a circle or an ellipse, then, in most cases, the second or third default filling method is used. If the operator, for some reason, decides to execute the first option by default, it is worth putting a horizontal line-by-line filling. Otherwise, the operator chooses the direction. If the selected area gravitates to a triangle, we act differently. Filling will always be parallel to one of the faces of the triangle. By default, select a face that is close to the horizontal. The problem is solved similarly with a rectangular shape. The filling will be parallel to one of the sides, which we select close to the vertical. If the rectangle has elongated proportions, then the filling is placed parallel to the longer side of the rectangle. This principle applies to any elongated figure. The final choice is left to the performer.

The equidistant method of forming the algorithm. The shape of the mosaic close to the circle is formed as follows (Fig. 9).

The distance between them is determined by the side of the teaser - $m$ and the distance between them - $p$. On each contour line $n_{i}$ we define points at a distance $d=m+p$ (Fig. 8).

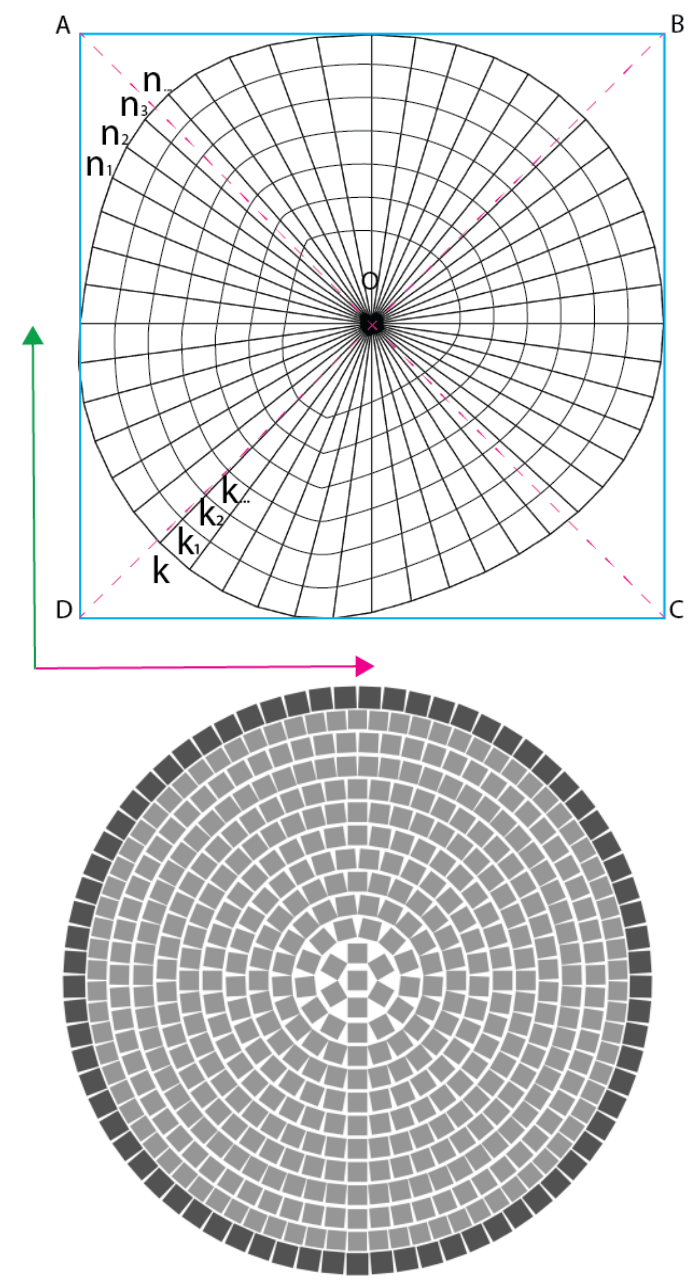

Fig. 9.

From each point we direct the vectors to the conditional center of the spot $-n_{i} O$. Further on these vectors we also determine the points $k_{i}$ as many times as possible at a distance $-d$. Connecting these points, we get the internal contours - $k_{1}, k_{2}, k_{3}, \ldots$.
When arranging teasers to form mosaics on concentric circles (Fig. 5), we use the following algorithm:

Determine the diameter of the circle on which we place the mosaic - $D$;

Choose the size of the side of the teaser $-m$ and the size of the seam - $p$;

Determine the distance between the centers of the teasers

$$
n=\frac{D}{2 \cdot d}
$$

Determine the average diameter of each row of concentric circles of each row

$$
D_{i}=\frac{\left(D_{\text {внеи. } i}+D_{\text {вн.i }}\right)}{2}
$$

Where is - $D_{\text {outi }}$ the outer diameter of each row;

$$
D_{\text {ini }} \text { - the inner diameter of each row; }
$$

Determine the number of teasers in each row

$$
k_{i}=\frac{D_{i} \cdot \pi}{d}
$$

We are building the mosaic.

Triangular and rectangular areas are filled in the same way.

If the spot of the mosaic is irregular in shape, choose the direction of the vectors along the normal to the external contour line at points that are located at a distance on the external contour $d$ (Fig. 10).

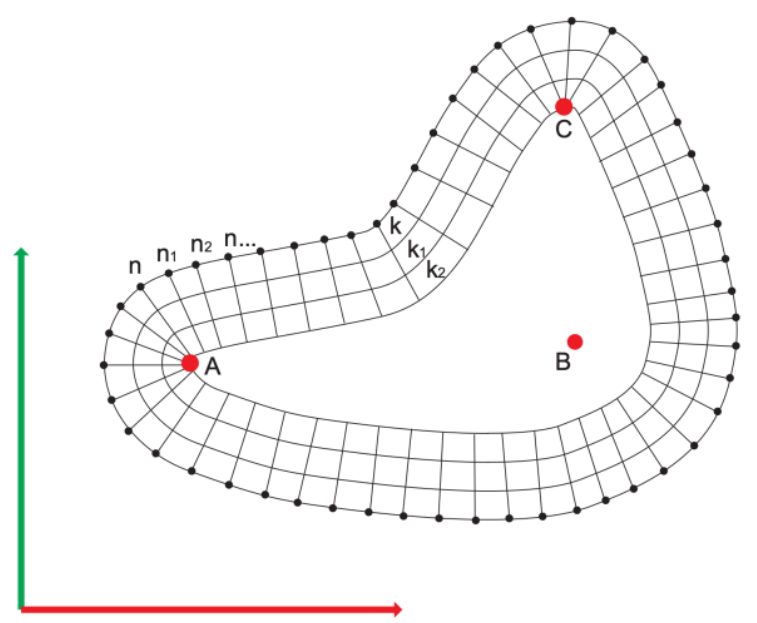

Fig. 10.

Inside the contour region, we obtain a set of intersection points of vectors. We find the shortest segment from the intersection point to the edge of the contour region. We take this distance as the base one and apply it to all vectors belonging to points on the selected path. On the resulting segments $n_{i} A$, we postpone as many times as possible to the distance $d$ and obtain the necessary points for the internal contours $k_{1}, k_{2}, k_{3}, \ldots$ To fill the remaining unfilled area in the center of the spot, you can use one of the above methods of filling, depending on the style.

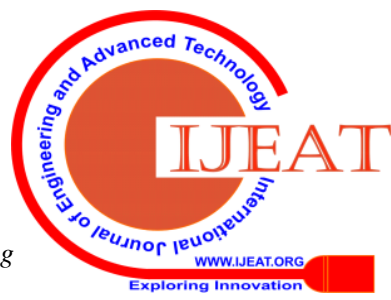


Spiral method of algorithm formation.

The spiral method of forming the algorithm for the contour region similar to a triangle, rectangle or square is similar to the previous ones. For contour regions of irregular shape, we can use the Held and Spielberger algorithm [8] to obtain spiral-like trajectories inside plane shapes bounded by line segments and circular arcs. As a parameter, the maximum transition distance specified by the user is used. The spiral vector guide starts in the inner part and ends at the borders of the selected contour region.

In fact, the technology of using vector guides is not limited only to these methods. Today, there are a large number of algorithms involved in filters for image processing. Many of these algorithms can be used to obtain an image or even a contour area filled with a vector pattern of a particular topology.

Such patterns can become the basis for peculiar patterns of mosaic fillings. This will no longer be the classical method, but the mosaic, like other forms of art, is subject to the influence of time and undergoes changes. Therefore, formal abstract solutions can be implemented using this technology. So far, we have only dealt with topology issues and have not raised color issues. The theory of color separation algorithms based on raster images has been developed in sufficient detail to date. If there is no finished color image, it is possible to create a color sketch based on the selected contour areas. In this case, the vector guide method can also be useful, since it fully corresponds to the method of creating color sketches in the traditional way.

\section{RESULT AND DISCUSSION}

As an example, consider the algorithm for filling a mosaic panel with a line-by-line and equidistant method.

When using the line-by-line method, we set the dimensions of the square-shaped panel $A=B=90$, the sizes of the sides of the teaser $-m=5$ and the seam $-p=1,425$, determine the distance between the centers of the teasers $d=m+p=6,425$ and the number of rows for the square

$$
n=\frac{A}{d}=\frac{90}{6,425}=14 \text {. }
$$

Number of teasers in a row for a square

$$
k=\frac{A}{d}=\frac{90}{6,425}=14 \text {. }
$$

The total number of teasers in the panel

$$
z=n \cdot k=14 \cdot 14=196 .
$$

The results are listed in Table I.

Table - I: Calculation Results for the line-by-line method

\begin{tabular}{|c|c|}
\hline Parameters & Result \\
\hline$A=B$ & 90 \\
\hline$m$ & 5,00 \\
\hline$p$ & 1,425 \\
\hline$d$ & 6,425 \\
\hline$n$ & 14 \\
\hline
\end{tabular}

$$
k_{i}=\frac{S_{i}}{d} .
$$

The total number of teasers in a round panel is

$$
z=\sum_{1}^{n} k_{i}=160 \text {. }
$$

The results are listed in Table II

Table - II: Calculation Results for the equidistant method

\begin{tabular}{|c|c|c|c|c|c|}
\hline $\boldsymbol{N}$ & $\boldsymbol{D}_{\text {out } \boldsymbol{i}}$ & $\boldsymbol{D}_{\text {in } \boldsymbol{i}}$ & $\boldsymbol{D i}$ & $\boldsymbol{S}_{\boldsymbol{i}}$ & $\boldsymbol{k}_{\boldsymbol{i}}$ \\
\hline 1 & 90 & 80 & 85,00 & 267,04 & 42 \\
\hline 2 & 77,15 & 67,15 & 72,15 & 226,67 & 35 \\
\hline 3 & 64,30 & 54,30 & 59,30 & 186,30 & 29 \\
\hline 4 & 51,45 & 41,45 & 46,45 & 145,93 & 23 \\
\hline 5 & 38,60 & 28,60 & 33,60 & 105,56 & 16 \\
\hline 6 & 25,75 & 15,75 & 20,75 & 65,19 & 10 \\
\hline 7 & 12,90 & 2,90 & 7,90 & 24,82 & 4 \\
\hline Center & & & & & 1 \\
\hline z & & & & & 160 \\
\hline
\end{tabular}

We are building the mosaic (Fig. 11). 


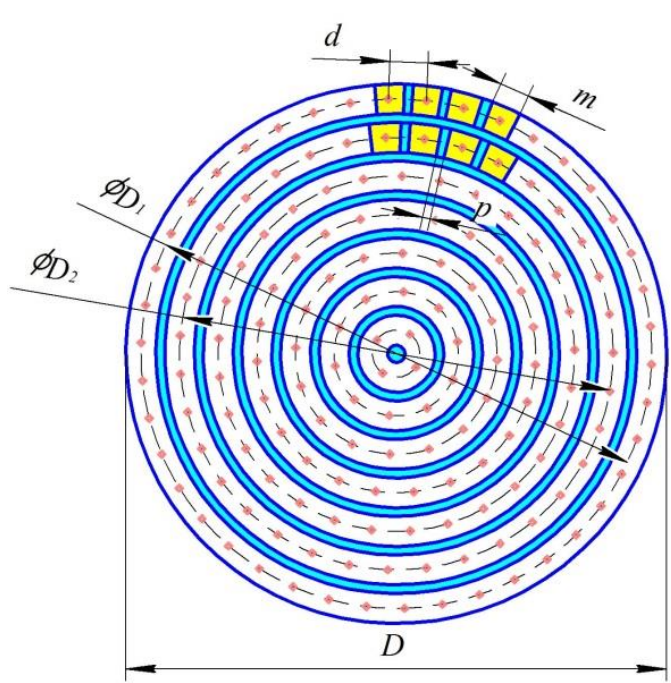

Fig. 11.

These algorithms allow you to create mosaic fillings based on linear patterns without color and tonal design, which is impossible for existing algorithms. The authors proposed the construction of the topology of classical mosaics using vector guides. Variants of using existing algorithms at each stage of the solution of the problem are proposed.

\section{CONCLUSION}

The proposed algorithms consist of three main stages:

1. Background filling using vector libraries or based on selected contour areas.

2. Contour rendering of the image in order to obtain the selected contour areas.

3. Filling closed contour areas using the following basic methods: a) line-by-line; b) equidistant; c) spiral.

It is possible to use a combination of these methods when forming a mosaic by an operator. Conclusion section is not required. Although a conclusion may review the main points of the paper, do not replicate the abstract as the conclusion. A conclusion might elaborate on the importance of the work or suggest applications and extensions.

\section{REFERENCES}

1. Hausner, A.: Simulating decorative mosaics. In Proceedings of SIGGRAPH 2001, pp. 573-580 (2001).

2. Fritzsche, L., Hellwig, H., Hiller, S., Deussen, O.: Interactive design of authenticlooking mosaics using voronoi structures. In: Proceedings of the 2nd InternationalSymposium on Voronoi Diagrams in Science and Engineering VD 2005 Conference,pp. 1-11 (2005)

3. D. de Ridder, P.W.Verbeek R.P.W. Duin, andL.J.vanVliet.The applicability of neural networks to non-linear image processing. Pattern Analysis and Applications, 2:111-128, 1999.

4. M. de Berg, O. Cheong, M. van Kreveld, and M. Overmars. Computational Geomet ry: Algorithms and applications. 3rd ed. Springer, 2008, isbn: $978-3-540$ - 77974- 2.

5. Battiato, S., Di Blasi, G., Gallo, G., Guarnera, G.C., Puglisi, G.: Artificial mosaics by gradient vector flow. In: Short Proceedings of Eurographics (2008).

6. Junhwan Kim, Fabio Pellacini Jigsaw Image Mosaics ACM Transicion on grafics 21(3):657-664 · July 2002.

7. Dong Hwa Kim, Ajith Abraham, and Jae Hoon Cho, A hybrid genetic algorithm and bacterial foraging approach for global optimization, Information Sciences, Volume 177, Issue 18, 15 September 2007, Pages 3918-3937.

8. Held, M. Spielberger, C. (2014). Improved spiral high-speed machining of multiply-connected pockets. Computer-Aided Design and Applications, 11(3), 346-357. J. Jones. (1991, May 10). Networks

\section{AUTHORS PROFILE}

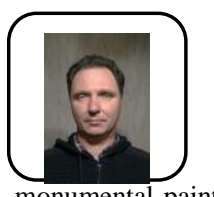

Andri Petrushevski. State university of infrastructure and technologies, Department of Information Technology, Kyiv, Ukraine, associate professor. Education: Ukrainian Academy of Arts and Architecture. Picturesque department. Workshop of monumental painting and temple culture of Professor M. A. Storozhenko. Master of Fine Arts. Institute of Computer Technology and digital graphics "Image Campus". 2007-2008 Argentina. Bs.As.

Worked as an designer in a company Creative Mind Group S.A. in Buenos Aires in 2002 - 2006. In 2016, he received a Ph.D. in technical sciences. More than 10 years of lecturer higher educational institutions in the city of Kiev (Ukraine). Research interests: theoretical development of visual graphics softwars for art, 3d Printing.

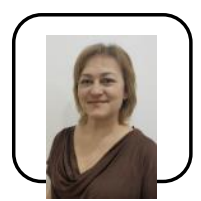

Victoriia Us. National Aviation University, Department of Interior Design, Kiev, Ukraine, Associate Professor. Education: graduated from Kiev National University of Construction and Architecture with a degree in architect, Department of Buildings and Structures. From 1994 to 2000 she worked as an architect in the company "Arch-Systems" in Kiev. Since 2000, a senior lecturer at universities in Kiev. She received her $\mathrm{PhD}$ in Technical Sciences in 2016. Scientific interests: composition and geometry when designing objects, decoration of sacred objects, interior and landscape design. 